Pacific Journal of Mathematics

AMENABILITY OF DISCRETE CONVOLUTION ALGEBRAS, 


\title{
AMENABILITY OF DISCRETE CONVOLUTION ALGEBRAS, THE COMMUTATIVE CASE
}

\author{
NiELS GRØNBEK
}

\begin{abstract}
A Banach algebra $\mathfrak{A}$ is called amenable if all bounded derivations into dual Banach $\mathfrak{A}$-modules are inner. Let $S$ be a semigroup and let $l^{1}(S)$ be the corresponding discrete convolution algebra. This paper is on the theme: "On the hypothesis that $l^{1}(S)$ is amenable, what conclusions can be drawn about the (algebraic) structure of $S$ ?" We give a complete characterization of commutative semigroups carrying amenable semigroup algebras. If $S$ is commutative, then $l^{1}(S)$ is amenable if and only if $S$ is a finite semilattice of groups, that is, there is a finite semilattice $Y$ and disjoint commutative groups $G_{\alpha}$ $(\alpha \in Y)$ such that $S=\bigcup_{\alpha \in Y} G_{\alpha}$ and $G_{\alpha} G_{\beta} \subseteq G_{\alpha \beta} \quad(\alpha, \beta \in Y)$.
\end{abstract}

The theme above has previously been studied in [3] and [4]. In both papers it is apparent that the condition of amenability imposes strong algebraic constraints on the semigroup. In [3] a rather complete description of inverse semigroups carrying amenable semigroup algebras is given. Of particular interest for this paper is that a semilattice carries an amenable semigroup algebra if and only if it is finite [3, Theorem 10]. In [4] it is proved that, if a one-sided cancellative semigroup carries an amenable semigroup algebra, then it is a group. The result of this paper, that for a commutative semigroup $S$, the semigroup algebra $l^{1}(S)$ is amenable if and only if $S$ is a finite lattice of groups, is proved by looking at the gross structure of $S$ by means of the "principle of maximal homomorphic image of a given type". Using the fact that homomorphic images of $S$ carry amenable semigroup algebras when $S$ does, we establish the necessity of the characterization by showing that each archimedean component of $S$ is a group. This is obtained by applying the results from [3] and [4], mentioned above, to the maximal semilattice, the maximal cancellative, and the maximal separative homomorphic images of $S$. The sufficiency of the characterization is easily verified. Alternatively, it follows from [3, Theorem 8].

1. Preliminaries. We shall need some elementary semigroup theory. We prefer to keep our exposition self-contained, so although most of what follows can be found in standard texts on the subject, 
we shall, with a few exceptions, give proofs in some detail. For a further discussion the reader is referred to [1]. Throughout $S$ will denote a commutative semigroup, with the binary operation written multiplicatively.

1.1. Definitions. Consider the following conditions on $S$ :

(A) Each element of $S$ is an idempotent.

(B) For all $s, t \in S$ there is $n \in \mathbf{N}$ such that

$$
s^{n} \in t S \text { and } t^{n} \in S S \text {. }
$$

(C) $s^{2}=t^{2}=s t \Rightarrow s=t \quad(s, t \in S)$.

If $S$ satisfies (A) we call $S$ a semilattice.

If $S$ satisfies (B) we call $S$ archimedean.

If $S$ satisfies (C) we call $S$ separative.

An ideal in $S$ is a subset $I$ such that $S I \subseteq I$. A prime ideal in $S$ is an ideal, whose complement is a subsemigroup of $S$.

A congruence on $S$ is an equivalence relation which is compatible with the semigroup operation.

A congruence $\sim$ on $S$ will be called separative (cancellative, archimedean, etc.) if the semigroup $S / \sim$ is separative (cancellative, archimedean, etc.).

1.2. Definition. (Principle of maximal homomorphic image of a given type). Let $\mathfrak{C}$ be a class of congruences on $S$, closed under intersections. Put $\rho_{0}=\bigcap\{\rho \mid \rho \in \mathfrak{C}\}$. Then $S / \rho_{0}$ is the maximal "type class $\mathfrak{C}$ " homomorphic image of $S$.

See also [1, p. 18] and $[7, \S 1]$.

EXAMPLE. Let $\rho_{0}=\bigcap\left\{\rho \mid s^{2} \rho s \quad(s \in S)\right\}$. Then $S / \rho_{0}$ is the maximal semilattice homomorphic image of $S$.

1.3. Definition. Let $s \in S$ and choose $m \in \mathbf{N}$ smallest possible so that $s^{m}=s^{m+r}$ for some $r \in \mathbf{N}$. Then $\operatorname{order}(s)=m$ and the smallest possible $r$ is called period $(s)$. If no such $m \in \mathbf{N}$ can be found we put $\operatorname{order}(s)=\infty$.

1.4. Definition. Let $S$ be a semigroup and suppose that there is a semilattice $Y$ and disjoint subsemigroups $S_{\alpha}(\alpha \in Y)$ of $S$ such that $S=\bigcup_{\alpha \in Y} S_{\alpha}$ and $S_{\alpha} S_{\beta} \subseteq S_{\alpha \beta} \quad(\alpha, \beta \in Y)$. Then $S$ is called a semilattice of the subsemigroups $S_{\alpha}(\alpha \in Y)$.

The following lemma is the main structure theorem for commutative semigroups. 
1.5. LemMA. Let $S$ be a commutative semigroup and let $Y$ be the maximal semilattice homomorphic image of $S$. Then there are disjoint archimedean subsemigroups $S_{\alpha}(\alpha \in Y)$ of $S$ such that $S$ is a semilattice of the semigroups $S_{\alpha}(\alpha \in Y)$. This decomposition of $S$ into archimedean subsemigroups is unique up to isomorphism of $Y$, and $S$ is separative if and only if each archimedean component $S_{\alpha}$ is cancellative.

Proof. See [1, §4.3].

1.6. Lemma. On $S$ define the relations:

$$
s c t \Leftrightarrow \exists u \in S s u=t u
$$

and

$$
s \sigma t \Leftrightarrow \exists n_{0} \in \mathbf{N} \forall n \geq n_{0} s^{n}=t^{n} .
$$

Then $c$ and $\sigma$ are congruences and $S / c$ is the maximal cancellative homomorphic image of $S$ and $S / \sigma$ is the maximal separative homomorphic image of $S$.

Proof. It is clear that both relations are congruences. Now suppose $\rho$ is a cancellative congruence; that is, $s u \rho t u \Rightarrow s \rho t \quad(s, t, u \in S)$. Then clearly $s c t \Rightarrow s \rho t \quad(s, t \in S)$ so that $c \subseteq \rho$. Since $c$ is cancellative we are done with the statements about $c$.

Now suppose that $s^{2} \sigma t^{2} \sigma s t$; that is, there is $n_{0} \in \mathbf{N}$ so that $s^{2 n}=$ $t^{2 n}=s^{n} t^{n}$ for $n \geq n_{0}$. Then $s^{4 n_{0}+1} t=s s^{2 n_{0}} \cdot t^{2 n_{0}} \cdot t=s^{2 n_{0}+1} t^{2 n_{0}+1}=$ $s^{4 n_{0}+2}$ so that for $n \geq 8 n_{0}+2$ we have $s^{n}=t^{n}$. Hence $s \sigma t$, proving that $\sigma$ is separative. Let $\rho$ be a separative congruence. If $s \sigma t$, then there is $k \in \mathbf{N}$ so that $s t^{k}=t^{k+1}$. In particular $s t^{k} \rho t^{k+1}$. This gives

$$
\left(s t^{k-1}\right)^{2}=s t^{k-2} s t^{k} \rho s t^{k-2} t^{k+1}=s t^{k-1} t^{k} \rho t^{k+1} t^{k-1}=\left(t^{k}\right)^{2} .
$$

With $x=s t^{k-1}$ and $y=t^{k}$ we have $x^{2} \rho y^{2} \rho x y$ so that $x \rho y$, that is, $s t^{k-1} \rho t^{k}$. Repeating as necessary, we get $s t \rho t^{2} \rho s^{2}$, where the second relation follows from symmetry. Thus $s \rho t$, proving that $\sigma \subseteq \rho$.

1.7. Lemma. $s^{2} \sigma s \Leftrightarrow \operatorname{order}(s)<\infty$ and $\operatorname{period}(s)=1$. If $e, f$ are idempotents in $S$, then $e \sigma f \Leftrightarrow e=f$.

Proof. Suppose $s^{2} \sigma s$. Then there is $n_{0} \in \mathbf{N}$ so that $s^{2 n}=s^{n}$ for $n \geq n_{0}$. If $r$ is the period of $s$ we have $2 n \equiv n(\bmod r)$ for $n \geq n_{0}$ so that $r=1$. The rest is obvious. 
1.8. LemMA. $S / \sigma$ is a group if and only if $S$ is archimedean with unique idempotent.

Proof. First suppose that $S / \sigma$ is a group. From Lemma 1.7 it follows that $S$ has a unique idempotent. Let $s, t \in S$. Since $S / \sigma$ is a group there are $u, v \in S$ so that $s u \sigma t$ and $t v \sigma s$. By definition of $\sigma, s$ divides a power of $t$ and $t$ divides a power of $s$, that is, $S$ is archimedean. Conversely, let $s \in S$ and let $e$ denote the unique idempotent in $S$. Since $S$ is archimedean there are $t, u \in S$ so that $s t=e$ and $u e=s^{n_{0}}$ for some $n_{0}$. We have $(e s)^{n_{0}+p}=e^{n_{0}+p} s^{n_{0}} s^{p}=$ $e^{n_{0}+p} u e s^{p}=u_{e s^{p}}=s^{n_{0}+p} \quad(p \in \mathbf{N})$ so that es $\sigma s$. Clearly st $\sigma e$, so $S / \sigma$ is a group.

2. The main theorem. For the remainder of this paper we shall assume that $S$ is a commutative semigroup such that $l^{1}(S)$ is amenable. We shall make frequent use of the fact that, if $T$ is a homomorphic image of $S$, then $l^{1}(T)$ is amenable, and if $I$ is an ideal in $S$ which is generated by an idempotent, then $l^{1}(I)$, being a closed $l^{1}(S)$-ideal which is unital as a Banach algebra, is amenable [6, Proposition 5.1]. Thus, if $S=\bigcup_{\alpha \in Y} S_{\alpha}$ is the decomposition of $S$ into its archimedean components, then the semilattice $Y$ is finite, since $l^{1}(Y)$ is amenable $([3$, Theorem 10$])$. We give $Y$ the usual semilattice ordering $\alpha \leq \beta \Leftrightarrow \alpha \beta=\alpha \quad(\alpha, \beta \in Y)$. Since $Y$ is finite, $Y$ has a minimal element, namely the product of all elements in $Y$.

It is convenient to start with the case where $S$ is separative; that is, we are assuming that each archimedean component is cancellative.

2.1. LeMmA. Let $S$ and $Y$ be as above and let $\alpha_{0}$ be the minimal element of $Y$. Then $S_{\alpha_{0}}$ is a group.

Proof. By [4, Theorem 2.3] $S / c$ is a group. Let $s \in S_{\alpha_{0}}$ Then there is $t \in S$ so that for all $u \in S$ stucu, that is, for all $u \in S$ there is $v \in S$ so that stuv $=u v$. Since $\alpha_{0}$ is minimal, st $\in S_{\alpha_{0}}$ and $u v \in S_{\alpha_{0}}$, so, using the cancellation law in $S_{\alpha_{0}}$, we see that $s t$ is a neutral element in $S_{\alpha_{0}}$. Consequently $l^{1}\left(S_{\alpha_{0}}\right)$ can be identified canonically with an ideal generated by an idempotent in $l^{1}(S)$. It follows that $l^{1}\left(S_{\alpha_{0}}\right)$ is amenable and therefore $S_{\alpha_{0}}$ is a group, again by $[4$, Theorem 2.3].

2.2. LEMMA. Let $l^{1}(S)$ be amenable and suppose that $S$ is separative. Then $S$ is a finite semilattice of groups. 
Proof. Let $S=\bigcup_{\alpha \in Y} S_{\alpha}$ be the decomposition of $S$ into its archimedean components. Let $\beta \in Y$, and define $T=\bigcup_{\alpha \geq \beta} S_{\alpha}$. Then $T$ is a subsemigroup of $S$ and $S \backslash T$ is a (prime) ideal in $S$. Hence the canonical Banach space direct sum $l^{1}(S)=l^{1}(T) \oplus l^{1}(S \backslash T)$ is a semidirect product, so that $l^{1}(T)$ is amenable. Since $\beta$ is minimal in $\{\alpha \in Y \mid \alpha \geq \beta\}$, Lemma 2.1 implies that $S_{\beta}$ is a group. But $\beta$ was arbitrary in $Y$.

We now turn to the general case.

2.3. Lemma. Suppose $l^{1}(S)$ is amenable. Then $S$ is a finite semilattice of its archimedean components, $S=\bigcup_{\alpha \in Y} S_{\alpha}$. Each $S_{\alpha}$ has a unique idempotent $e_{\alpha}$, and $e_{\alpha} S_{\alpha}$ is a group, isomorphic to the maximal separative homomorphic image of $S_{\alpha}$.

Proof. By Lemma $2.2 S / \sigma$ is a finite semilattice of groups, $S / \sigma=$ $\bigcup_{\alpha \in Y} G_{\alpha}$. Let $S_{\alpha}$ be the preimage of $G_{\alpha}$ by the canonical map $S \rightarrow S / \sigma$. With slight abuse of notation we have $S_{\alpha} / \sigma=G_{\alpha}$, so that $S_{\alpha}$ is archimedean with unique idempotent, $e_{\alpha}$ say, by Lemma 1.8. It follows that $S=\bigcup_{\alpha \in Y} S_{\alpha}$ is the decomposition of $S$ into its archimedean components. Now let $s \in S_{\alpha}$. Since $G_{\alpha}$ is a group, there is $t \in S_{\alpha}$ so that $s t \sigma e_{\alpha}$, i.e. $(s t)^{n}=e_{\alpha}$ for some $n \in \mathbf{N}$. Hence $e_{\alpha} s^{n-1} t^{n}$ is an inverse to $e_{\alpha} s$. Clearly the canonical map from $e_{\alpha} S_{\alpha}$ to $G_{\alpha}$ is surjective. Assume that $e_{\alpha} s \sigma e_{\alpha}$ for some $s \in S_{\alpha}$. Since $e_{\alpha} S_{\alpha}$ is a group it follows from Lemma 1.7 that $e_{\alpha} s=e_{\alpha}$, proving injectivity of the canonical map.

We shall finish the proof of the main theorem by proving that $e_{\alpha} S_{\alpha}=S_{\alpha}$ for each $\alpha \in Y$. This is done by exploiting that $l^{1}(S)$, being amenable, has a bounded approximate identity. First we need a definition.

2.4. Definition. Let $s \in S$. Then we define

$$
\left[s s^{-1}\right]=\{u \in S \mid u s=s\} .
$$

Since $l^{1}(S)$ has a bounded approximate identity $\left[s s^{-1}\right] \neq \varnothing$ for all $s \in S[4$, Theorem 1.1].

2.5. Lemma. Let $S=\bigcup_{\alpha \in Y} S_{\alpha}$ be the decomposition of $S$ into its archimedean components, as in Lemma 2.3, and let $s \in S_{\alpha}$. If $\left[s s^{-1}\right] \cap S_{\alpha} \neq \varnothing$, then $s \in e_{\alpha} S_{\alpha}$. If $\alpha$ is maximal in $Y$, then $S_{\alpha}$ is a group. 
Proof. Let $u \in\left[s s^{-1}\right] \cap S_{\alpha}$. Then $u s \sigma e_{\alpha} s$. Since $S_{\alpha} / \sigma$ is a group we have $u \sigma e_{\alpha}$, i.e. $u^{n}=e_{\alpha}$ for some $n \in \mathbf{N}$. Hence $s=u^{n} s=e_{\alpha} s$. In general, if $s \in S_{\alpha}$ and $u \in\left[s s^{-1}\right] \cap S_{\beta}$, then $s=u s \in S_{\alpha} \cap S_{\beta \alpha}$, so $\beta \geq \alpha$. Thus, when $\alpha$ is maximal in $Y$ we have that $\left[s s^{-1}\right] \subseteq S_{\alpha}$ for all $s \in S_{\alpha}$. It follows that $e_{\alpha} S_{\alpha}=S_{\alpha}$, so that $S_{\alpha}$ is a group by Lemma 2.3.

2.6. Lemma. Let $s=\bigcup_{\alpha \in Y} S_{\alpha}$ be as in Lemma 2.3. Then [ss $\left.{ }^{-1}\right] \cap$ $\left\{e_{\alpha} \mid \alpha \in Y\right\} \neq \varnothing$ for all $s \in S$. In particular $l^{1}(S)$ is unital.

Proof. First note that, if $u \in\left[s s^{-1}\right]$, then $\left[u u^{-1}\right] \subseteq\left[s s^{-1}\right]$. Let $s \in S$ and let $S_{\alpha_{0}}$ be the archimedean component of $s$. Put $u_{0}=s$ and choose successively $u_{k} \in\left[u_{k-1} u_{k-1}^{-1}\right]$. Let $S_{\alpha_{k}}$ be the archimedean component of $u_{k}$. As noted in the proof of Lemma 2.5 we have $\alpha_{0} \leq \alpha_{1} \leq \cdots \leq \alpha_{k} \leq \cdots$. Since card $Y<\infty$, we eventually have $S_{\alpha_{k}}=S_{\alpha_{k+1}}$, whence $\left[u_{k} u_{k}^{-1}\right] \cap S_{\alpha_{k}} \neq \varnothing$, so that $e_{\alpha_{k}} \in\left[u_{k} u_{k}^{-1}\right]$ by Lemma 2.5. As observed in the beginning of the proof $e_{\alpha_{k}} \in\left[s s^{-1}\right]$. From [5, Theorem 7.5] it follows that $l^{1}(S)$ has a unit.

We are now able to prove:

2.7. TheOREM. Let $S$ be a commutative semigroup. Then $l^{1}(S)$ is amenable if and only if $S$ is a finite semilattice of commutative groups.

Proof. The sufficiency has been noted in the introduction. Hence we assume that $l^{1}(S)$ is amenable. Let $s=\bigcup_{\alpha \in Y} S_{\alpha}$ be the decomposition as in Lemma 2.3. By Lemma 2.5 the theorem is true if $\operatorname{card} Y=1$. We proceed by induction on $n=\operatorname{card} Y$. Assume that $n \geq 2$ and that the theorem is true for semigroups which are semilattices of archimedean semigroups with cardinality of the semilattice strictly less than $n$. Let $\alpha_{0}$ be the minimal element in $Y$. Let $\beta \in Y \backslash\left\{\alpha_{0}\right\}$, and define $T_{\beta}=\bigcup_{\alpha \geq \beta} S_{\alpha}$. As in the proof of Lemma 2.2, we see that $l^{1}\left(T_{\beta}\right)$ is amenable. Thus, by the induction hypothesis, we have that $S_{\alpha}$ is a group for $\alpha \in Y \backslash\left\{\alpha_{0}\right\}$. We finish the induction step by proving that $S_{\alpha_{0}}=e_{\alpha_{0}} S_{\alpha_{0}}$. To this end, define a congruence $\sim$ on $S$ by

$$
s \sim t \Leftrightarrow S s=S t \quad(s, t \in S) .
$$

Note that, if $s \sim t$, then $s \in S t$, since $\left[s s^{-1}\right] \neq \varnothing$. Using that $S_{\alpha}$ is a group for $\alpha \neq \alpha_{0}$, we see that $S / \sim \cong \bigcup_{\alpha \neq \alpha_{0}}\left\{e_{\alpha}\right\} \cup S_{\alpha_{0}} / \sim$. Hence $l^{1}\left(S_{\alpha_{0}} / \sim\right)$ is (isomorphic to) a closed ideal of finite codimension in the 
amenable Banach algebra $l^{1}(S / \sim)$, and therefore $l^{1}\left(S_{\alpha_{0}} / \sim\right)$ is itself amenable [2, Theorem 4.1]. From Lemma 2.5 we get that $S_{\alpha_{0}} / \sim$ is a group. In particular we have for all $s \in S_{g a_{0}}$ that $s \sim e_{\alpha_{0}} s$, so, by the note above, $S_{\alpha_{0}} \subseteq e_{\alpha_{0}} S_{\alpha_{0}}$. The induction step is hereby completed.

Acknowledgment. I wish to acknowledge a stimulating correspondence with Professor J. Duncan on the subject. I wish to thank Dr. K. B. Laursen for a careful reading of the manuscript.

\section{REFERENCES}

[1] A. H. Clifford and G. B. Preston, The algebraic theory of semigroups, Math. Surveys no. 7, Amer. Math. Soc., Providence, Rhode Island 1967.

[2] P. C. Curtis and R. J. Loy, The structure of amenable Banach algebras; (to appear in J. London Math. Soc.).

[3] J. Duncan and I. Namioka, Amenability of inverse semigroups and their semigroup algebras, Proc. Royal Soc. Edinburgh, 80A (1978), 309-321.

[4] N. Grønbæk, Amenability of weighted discrete convolution algebras on cancellative semigroups, Proc. Royal Soc. Edinburgh, 110A (1988), 351-360.

[5] E. Hewitt and H. S. Zuckerman, The $l_{1}$-algebra of a commutative semigroup, Trans. Amer. Math. Soc., 83 (1956), 70-97.

[6] B. E. Johnson, Cohomology in Banach algebras; Mem. Amer. Math. Soc., 127 (1972).

[7] T. Tamura, The study of closets and free contents related to semilattice decomposition of semigroups; in Semigroups, Proceedings of a symposium on semigroups held at Wayne State University, Detroit, Michigan, (1968); Academic Press, New York and London.

Received August 29, 1988.

Københavns Universitets Matematiske Institut

UNIVERSITETSPARKEN 5

2100 København $\varnothing$, Denmark 



\section{PACIFIC JOURNAL OF MATHEMATICS EDITORS}

\author{
V. S. VARADARAJAN \\ (Managing Editor) \\ University of California \\ Los Angeles, CA 90024-1555-05 \\ Herbert Clemens \\ University of Utah \\ Salt Lake City, UT 84112 \\ ThOMAs ENRIGHT \\ University of California, San Diego \\ La Jolla, CA 92093
}

R. FINN

Stanford University

Stanford, CA 94305

HeRmanN FlaschKa

University of Arizona

Tucson, AZ 85721

VAUGHAN F. R. JoNES

University of California

Berkeley, CA 94720

SteVen KerCKhofF

Stanford University

Stanford, CA 94305
ROBION KIRBY

University of California

Berkeley, CA 94720

C. C. Moore

University of California

Berkeley, CA 94720

HAROLD STARK

University of California, San Diego

La Jolla, CA 92093

\section{ASSOCIATE EDITORS}
R. ARENS
E. F. BECKENBACH
B. H. NeumanN
F. Wolf
K. YoshidA
(1906-1982)
(1904-1989)

\section{SUPPORTING INSTITUTIONS}

UNIVERSITY OF ARIZONA
UNIVERSITY OF BRITISH COLUMBIA
CALIFORNIA INSTITUTE OF TECHNOLOGY
UNIVERSITY OF CALIFORNIA
MONTANA STATE UNIVERSITY
UNIVERSITY OF NEVADA, RENO
NEW MEXICO STATE UNIVERSITY
OREGON STATE UNIVERSITY
UNIVERSITY OF ARIZONA
UNIVERSITY OF BRITISH COLUMBIA
UNIVERSITY OF CALIFORNIA
MONTANA STATE UNIVERSITY
NEW MEXICO STATE UNIVERSITY

\author{
UNIVERSITY OF OREGON \\ UNIVERSITY OF SOUTHERN CALIFORNIA \\ STANFORD UNIVERSITY \\ UNIVERSITY OF HAWAII \\ UNIVERSITY OF TOKYO \\ UNIVERSITY OF UTAH \\ WASHINGTON STATE UNIVERSITY \\ UNIVERSITY OF WASHINGTON
}

The Supporting Institutions listed above contribute to the cost of publication of this Journal, but they are not owners or publishers and have no responsibility for its content or policies.

Mathematical papers intended for publication in the Pacific Journal of Mathematics should be in typed form or offset-reproduced (not dittoed), double spaced with large margins. Please do not use built up fractions in the text of the manuscript. However, you may use them in the displayed equations. Underline Greek letters in red, German in green, and script in blue. The first paragraph must be capable of being used separately as a synopsis of the entire paper. In particular it should contain no bibliographic references. Please propose a heading for the odd numbered pages of less than 35 characters. Manuscripts, in triplicate, may be sent to any one of the editors. Please classify according to the 1980 Mathematics Subject Classification (1985 Revision) scheme which can be found in the December index volumes of Mathematical Reviews. Supply name and address of author to whom proofs should be sent. All other communications should be addressed to the managing editor, or Elaine Barth, University of California, Los Angeles, California 90024-1555-05.

There are page-charges associated with articles appearing in the Pacific Journal of Mathematics. These charges are expected to be paid by the author's University, Government Agency or Company. If the author or authors do not have access to such Institutional support these charges are waived. Single authors will receive 50 free reprints; joint authors will receive a total of 100 free reprints. Additional copies may be obtained at cost in multiples of 50 .

The Pacific Journal of Mathematics is issued monthly as of January 1966. Regular subscription rate: $\$ 190.00$ a year (6 Vols., 12 issues). Special rate: $\$ 95.00$ a year to individual members of supporting institutions.

Subscriptions, orders for numbers issued in the last three calendar years, and changes of address should be sent to Pacific Journal of Mathematics, P.O. Box 969, Carmel Valley, CA 93924, U.S.A. Old back numbers obtainable from Kraus Periodicals Co., Route 100, Millwood, NY 10546.

The Pacific Journal of Mathematics at P.O. Box 969, Carmel Valley, CA 93924 (ISSN 0030-8730) is published monthly. Second-class postage paid at Carmel Valley, California 93924, and additional mailing offices. Postmaster: send address changes to Pacific Journal of Mathematics, P.O. Box 969, Carmel Valley, CA 93924.

\section{PUBLISHED BY PACIFIC JOURNAL OF MATHEMATICS, A NON-PROFIT CORPORATION}




\section{Pacific Journal of Mathematics}

\section{Vol. 143, No. $2 \quad$ April, 1990}

Gustavo Corach, Horacio Porta and Lázaro Recht, Differential geometry of systems of projections in Banach algebras ................. 209

Peter Fleischmann and Jens Carsten Jantzen, Simple periodic modules of



Niels Gronbaek, Amenability of discrete convolution algebras, the commutative case ...................................243

Nguyên H. V. Hung, The mod 2 equivariant cohomology algebras of

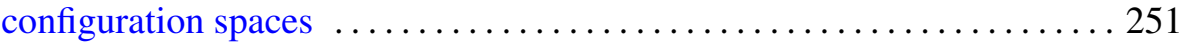

Wojciech Kucharz, Global almost analytic algebraicity of analytic sets . . . 287 John Merrill, A class of consistent anti-Martin's axioms .............. 301

Takafumi Murai, The power 3/2 appearing in the estimate of analytic

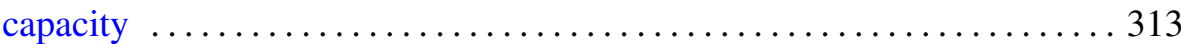

L. Panaitopol and Doru Stefanescu, On the generalized difference

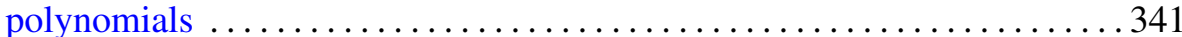

Katsuhiro Shiohama, Takashi Shioya and Minoru Tanaka, Mass of rays



Gerhard Ströhmer, About compressible viscous fluid flow in a bounded

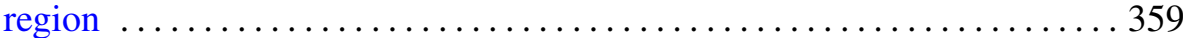

A. Ülger, Arens regularity sometimes implies the RNP 